\title{
Keeping qRT-PCR rigorous and biologically relevant
}

\author{
John Bennett · David Hondred · James C. Register III
}

Received: 30 September 2014 / Accepted: 1 October 2014/Published online: 11 October 2014

(C) Springer-Verlag Berlin Heidelberg 2014

\section{Introduction}

Because of the importance of differential gene expression in the growth, development, and reproduction of organisms, assays of mRNA transcript levels are widely employed in biological research. The most frequently used assay combines reverse transcription (RT) with quantitative polymerase chain reaction (qPCR) and is known as qRT-PCR (or RT-qPCR). In practice, RT generates a single-stranded cDNA copy of each molecule in an mRNA population, after which a qPCR thermocycler is used in conjunction with gene-specific oligonucleotide primers to amplify selected target cDNAs (Bustin et al. 2009). Fluorescent probes allow progress to be monitored through three phases: an initial phase during which the increasing signal cannot be distinguished from background noise, a 2nd phase of observable exponential signal increase, and a third and final phase of slowing signal increase as reagents and other factors become rate limiting. Quantification of each target mRNA requires the determination of the number of cycles (known as $\mathrm{Ct}, \mathrm{Cq}$, $\mathrm{Cp}$, or TOP) required to reach a pre-set fluorescence threshold during the second phase. When a transcript is twice as abundant in one sample as it is in another, the $\mathrm{Ct}$

Communicated by Neal Stewart.
J. Bennett
Colombo, Sri Lanka
e-mail: jbennett@irrialumni.org
D. Hondred · J. C. Register III ( $\square)$
DuPont Pioneer, 7300 NW 62nd Ave, Johnston, IA 50131, USA
e-mail: jim.register@ pioneer.com
D. Hondred
e-mail: david.hondred@pioneer.com

will occur about one cycle earlier for the more abundant transcript. The varying transcript levels of target genes must then be normalized against the stable transcript levels of one or more reference genes present in the same mRNA population. Algorithms are available to validate the stability of these reference genes. With use of appropriate controls, standardization, and normalization, measurements of $\mathrm{Ct}$ can lead to reliable estimates of the relative amount of a target transcript in the input mRNA population (Bustin et al. 2009).

Despite its complexity, qRT-PCR is rapid and cost effective, and, if sufficient care is taken with the design and execution of experiments, it is reliable and sensitive. There are, however, two trends of concern in the publication of qRT-PCR experiments. The first is that many papers do not contain enough information to judge whether the data are reliable. Bustin et al. (2009) addressed this question by defining "minimum information for publication of quantitative real-time PCR experiments" (MIQE). The second trend of concern is the increasing number of manuscripts that claim to have identified reference genes without comparing them to target genes in a well-defined and relevant biological context. We agree with Bustin et al. (2010), who considered it pointless and misleading to publish such 'reference gene papers' as stand-alone reports.

In response to these trends, we propose that publishable qRT-PCR manuscripts should satisfy two criteria. First, the design, execution, and reporting of qRT-PCR experiments should address the items listed in the MIQE guidelines. Second, the selection and validation of reference genes should be reported in relation to target gene behavior in a well-defined biological context. Here we discuss the background to these criteria and their implementation relative to plant biology studies. 


\section{The MIQE checklist}

The MIQE checklist provided in Table 1 of Bustin et al. (2009) and in précis form in Table 1 of Bustin et al. (2010) identifies a large number of parameters essential or desirable in the conduct and reporting of reliable high-quality qPCR and qRT-PCR experiments. The checklists include topics from experimental design to execution to data analysis and interpretation. Because these topics are interrelated, they should be approached holistically during experimental design. Bustin et al. (2009) advocated submission of the completed checklist along with the manuscript and then, if the manuscript is accepted, publishing it online as part of the supplemental information available to readers.

From 2009 to mid-2014, about 200 plant publications cited the MIQE guidelines, but only about $10 \%$ included some form of completed checklist in a supplement. A good example of a completed MIQE checklist is Additional File 4 of Fujisawa et al. (2011) on fruit ripening in tomato. At first glance, the checklist may appear intimidating, but it is clearly widely applicable and in a format that is conducive to the gradual accumulation of data as qRT-PCR experiments are planned, conducted, and analyzed before being reported and peer reviewed.

A publication that does not provide a MIQE checklist but is nevertheless clearly inspired by the MIQE guidelines is the work of de Keyser et al. (2013). It focuses on the flavonoid pigment biosynthetic pathway in azalea (Rhododendron simsii hybrids) and illustrates methods for checking quantity, purity, and integrity of mRNA; primer design; estimating qPCR efficiency; and selecting, validating, and optimizing the number of reference genes. The authors employ the reference genes to normalize six genes of the flavonoid pathway with the geNorm software of Vandesompele et al. (2002).

Two additional issues are the efficiency of RT and the avoidance of primer dimers, which can contribute to background fluorescence. These issues were addressed by Saha and Blumwald (2014) in their study of expression patterns for both endogenous genes and transgenes in pearl millet (Pennisetum glaucum). This publication provides an excellent tutorial on qRT-PCR, while not referring to MIQE. The authors employed geNorm and five other software packages (Stability Index, Normfinder, $\Delta \mathrm{Ct}$, BestKeeper, RefFinder) to validate reference genes for use in normalization.

\section{Reference genes}

As qRT-PCR has matured, so has thinking about the selection, validation, and use of reference genes. In the infancy of qRT-PCR, researchers often chose a single "housekeeping" gene for normalization of target genes, but the expression of "housekeeping" genes can be highly variable. Best practices today call for the use of at least 3 reference genes that are demonstrated to be expressed stably under the same experimental conditions used to study expression of the target genes (Hruz et al. 2011) and at abundance levels similar to those of the target genes (Czechowski et al. 2005).

Novel reference genes are most easily identified in plant species for which extensive gene expression databases are already available. The model plant Arabidopsis thaliana, many cereals, and commercial crops such as soybean and cotton have these resources. Czechowski et al. (2005) and Hruz et al. (2011) provided details about identifying novel reference genes for A. thaliana from microarray databases. For plant species that lack these resources, other approaches can be effective. In the case of azalea, de Keyser et al. (2013) found an azalea EST library to be adequate. Saha and Blumwald (2014) found that foxtail millet formed a bioinformatic bridge between pearl millet and rice, enabling homologues of reference genes validated in rice to be found in foxtail millet and then in pearl millet, where validation was confirmed.

\section{Concluding remarks}

MIQE has received support from journals, authors and suppliers of equipment, reagents, and software (http://miqepress.gene-quantification.info/). De Keyser et al. (2013) and Saha and Blumwald (2014) proved that qRT-PCR data can be rigorously conducted and reported without inclusion of a MIQE checklist. However, we do advocate that the items on the checklist be addressed during experimental design and execution and recommend that a checklist be made available to referees during the review process. Except for rare and justified cases (Bustin et al. 2010), reference genes should be published in conjunction with their use in normalizing target genes within a biological context. Finally, we emphasize that, for every manuscript that includes qRT-PCR data, it is vital that authors give careful attention to the qRT-PCR experimental details to ensure that gene expression data from this powerful method are valid.

Conflict of interest The views expressed in this article are those of David Hondred and James C. Register and do not necessarily represent the views of, and should not be attributed to, DuPont Pioneer.

\section{References}

Bustin SA, Benes V, Garson JA, Hellemans JA, Huggett J, Kubista M, Mueller R, Nolan T, Pfaffl MW, Shipley GL, Vandesompele 
J, Wittwer CT (2009) The MIQE guidelines: minimum information for publication of quantitative real-time PCR experiments. Clin Chem 55:611-622

Bustin SA, Beaulieu JF, Huggett J, Jaggi R, Kibenge FSB, Olsvik PA, Penning LC, Toegel S (2010) MIQE précis: practical implementation of minimum standard guidelines for fluorescencebased quantitative real-time PCR experiments. BMC Mol Biol $11: 74$

Czechowski T, Stitt M, Altmann T, Udvardi MK, Scheible W-R (2005) Genome-wide identification and testing of superior reference genes for transcript normalization in Arabidopsis. Plant Physiol 139:5-17

De Keyser E, Desmet L, Van Bockstaele E, De Riek J (2013) How to perform RT-qPCR accurately in plant species? A case study on flower colour gene expression in an azalea (Rhododendron simsii hybrids) mapping population. BMC Mol Biol 14:13
Fujisawa M, Nakano T, Ito Y (2011) Identification of potential target genes for the tomato fruit-ripening regulator RIN by chromatin immunoprecipitation. BMC Plant Biol 11:26

Hruz T, Wyss M, Docquier M, Pfaffl MW, Masanetz S, Borghi L, Verbrugghe P, Kalaydjieva L, Bleuler S, Laule O, Descombes P, Gruissem W, Zimmerman P (2011) RefGenes: identification of reliable and condition specific reference genes for RT-qPCR data normalization. BMC Genom 12:156

Saha P, Blumwald E (2014) Assessing reference genes for accurate transcript normalization using quantitative real-time PCR in pearl millet [Pennisetum glaucum (L.) R. Br]. PLoS ONE 9:e106308

Vandesompele J, De Preter K, Pattyn F, Poppe B, Van Roy N, De Paepe A, Speleman F (2002) Accurate normalization of real-time quantitative RT-PCR data by geometric averaging of multiple internal control genes. Genome Biol 3:1-11 